\title{
THE ANISOTROPIC XY MODEL ON THE 1D ALTERNATING SUPERLATTICE
}

\author{
F. F. Barbosa Filho ${ }^{1}$, J. P. de Lima ${ }^{1}$ and L. L. Gonçalves ${ }^{2}$ \\ ${ }^{1}$ Departamento de Física da UFPi, Campus do Ininga, 64049-550 Teresina, \\ Piauí, Brazil \\ ${ }^{2}$ Departamento de Física da UFC, Campus do Pici, C.P. 6030, 60451-970 \\ Fortaleza, Ceará, Brazil
}

\begin{abstract}
The anisotropic XY-model in a transverse field $(s=1 / 2)$ on the onedimensional alternating superlattice (closed chain) is considered. The solution of the model is obtained by introducing a generalized Jordan-Wigner transformation which maps the system onto a non-interacting fermion gas. The exact excitation spectrum is determined by reducing the problem to a diagonalization of a block matrix, and it is shown that it is numerically identical to the one obtained by using the approximate transfer matrix method . The induced magnetization and the susceptibility $\chi^{z z}$ are determined as a function of the transverse field, and it is shown that, at $T=0$, the susceptibility presents multiple singularities. It is also shown, as expected, that this critical behaviour driven by transverse field belongs to the same universality class of the model on the alternating chain.
\end{abstract}

Keywords: xy-model, one-dimensional superlattice, quantum transitions Corresponding author: J. P. de Lima, Departamento de Física da UFPi, Campus do Ininga,64049-550Teresina, Piauí, Brazil

mentel@mnnet.com.br)

(FAX: +55-86-215-5560; e-mail: pi- 
We have recently considered the isotropic XY model on an alternating superlattice $^{1}$, which we have been able to solve exactly. Although the model presents a critical behaviour, this behaviour is related to the induced magnetization since, even at $\mathrm{T}=0$, it does not present spontaneous magnetization ${ }^{2}$. Therefore, in order to study the critical behaviour induced by the transverse field when there is spontaneous order in the ground state, we will consider the anisotropic XY model on the one-dimensional alternating superlattice $(s=1 / 2)$. The Hamiltonian of the system is given by

$$
\begin{aligned}
H= & -\frac{1}{2} \sum_{l=1}^{N}\left\{\sum_{m=1}^{n_{A}} 2 h_{A} S_{l, m}^{A^{Z}}+\sum_{m=1}^{n_{B}} 2 h_{B} S_{l, m}^{B^{Z}}+\right. \\
& +\left\{\sum_{m=1}^{n_{A}-1} J_{A}\left(S_{l, m}^{A^{+}} S_{l, m+1}^{A^{-}}+\gamma_{A} S_{l, m}^{A^{+}} S_{l, m+1}^{A^{+}}\right)+\right. \\
& +\sum_{m=1}^{n_{B}-1} J_{B}\left(S_{l, m}^{B^{+}} S_{l, m+1}^{B^{-}}+\gamma_{B} S_{l, m}^{B^{+}} S_{l, m+1}^{B^{+}}\right)+ \\
& +J\left[\left(S_{l, n_{A}}^{A^{+}} S_{l, 1}^{B^{-}}+S_{l, n_{B}}^{B^{+}} S_{l+1,1}^{A^{-}}\right)+\right. \\
& \left.\left.\left.+\gamma\left(S_{l, n_{A}}^{A^{+}} S_{l, 1}^{B^{+}}+S_{l, n_{B}}^{B^{+}} S_{l+1,1}^{A^{+}}\right)\right]\right\}+ \text {h.c. }\right\}
\end{aligned}
$$

where $S^{ \pm}=S^{X} \pm i S^{Y}, J$ and $\gamma$ are respectively the exchange and anisotropy parameters between spins at the interfaces, $J_{A}\left(J_{B}\right)$ and $\gamma_{A}\left(\gamma_{B}\right)$ the exchange and anisotropy parameters for spins within the subcell $A(B)$, and $h_{A}\left(h_{B}\right)$ is the transverse field in the medium $A(B)$. The spin operators can be expressed in terms of fermion operators using the generalized Jordan-Wigner ${ }^{3}$

$$
\begin{aligned}
& S_{l, m}^{A^{+}}=\exp \left\{i \pi \sum_{n=1}^{l-1} \sum_{r=1}^{n_{A}} a_{n, r}^{\dagger} a_{n, r}+i \pi \sum_{r=1}^{m-1} a_{l, r}^{\dagger} a_{l, r}+i \pi \sum_{n=1}^{l-1} \sum_{r=1}^{n_{B}} b_{n, r}^{\dagger} b_{n, r}\right\} a_{l, m}^{\dagger}, \\
& S_{l, m}^{B^{+}}=\exp \left\{i \pi \sum_{n=1}^{l} \sum_{r=1}^{n_{A}} a_{n, r}^{\dagger} a_{n, r}+i \pi \sum_{r=1}^{m-1} b_{l, r}^{\dagger} b_{l, r}+i \pi \sum_{n=1}^{l-1} \sum_{r=1}^{n_{B}} b_{n, r}^{\dagger} b_{n, r}\right\} b_{l, m}^{\dagger},
\end{aligned}
$$

and by introducing this transformation in Eq.(1), the Hamiltonian can be written in the form 


$$
\begin{array}{ll}
H= & -\sum_{l=1}^{N}\left\{\sum_{m=1}^{n_{A}} h_{A} a_{l, m}^{\dagger} a_{l, m}+\sum_{m=1}^{n_{B}} h_{B} b_{l, m}^{\dagger} b_{l, m}+\right. \\
+ & \sum_{m=1}^{n_{A}-1} \frac{J_{A}}{2}\left(a_{l, m}^{\dagger} a_{l, m+1}+\gamma_{A} a_{l, m}^{\dagger} a_{l, m+1}^{\dagger}+\text { h.c. }\right)+ \\
+\quad & \sum_{m=1}^{n_{B}-1} \frac{J_{B}}{2}\left(b_{l, m}^{\dagger} b_{l, m+1}+\gamma_{B} b_{l, m}^{\dagger} b_{l, m+1}^{\dagger}+h . c .\right)+ \\
+\quad & \left.\frac{J}{2}\left[a_{l, n_{A}}^{\dagger} b_{l, 1}+b_{l, n_{B}}^{\dagger} a_{l+1,1}+\gamma\left(a_{l, n_{A}}^{\dagger} b_{l, 1}^{\dagger}+b_{l, n_{B}}^{\dagger} a_{l+1,1}^{\dagger}\right)+h . c .\right]\right\}+ \\
+\quad & \frac{N\left(n_{A} h_{A}+n_{B} h_{B}\right)}{2},
\end{array}
$$

where $a$ 's e b's are fermion operators, and we have neglected a boundary term which, in the thermodynamic limit, will not give any contribution for the static properties. Then, introducing in Eq. (4) the Fourier transforms

$$
a(b)_{l j}=\frac{1}{\sqrt{N}} \sum_{Q} e^{-i Q d l} A(B)_{Q, j},
$$

where $Q=2 \pi n / N d, n=1,2,3, \ldots, N$ and $d=n_{A}+n_{B}$ is the size of the cell, the Hamiltonian can be written as $H=-\sum_{Q} H_{Q}$, where

$$
\begin{aligned}
H_{Q}= & \sum_{m=1}^{n_{A}-1} h_{A} A_{Q, m}^{\dagger} A_{Q, m}+\sum_{m=1}^{n_{B}-1} h_{B} B_{Q, m}^{\dagger} B_{Q, m}+ \\
& \sum_{m=1}^{n_{A}-1}\left(\frac{J_{A}}{2} A_{Q, m}^{\dagger} A_{Q, m+1}+\gamma_{A} A_{Q, m}^{\dagger} A_{-Q, m+1}^{\dagger}+h . c\right)+ \\
& \sum_{m=1}^{n_{B}-1}\left(\frac{J_{B}}{2} B_{Q, m}^{\dagger} B_{Q, m+1}+\gamma_{B} B_{Q, m}^{\dagger} B_{-Q, m+1}^{\dagger}+h . c\right)+ \\
& \frac{J}{2}\left[A_{Q, n_{A}}^{\dagger} B_{Q, 1}+B_{Q, n_{B}}^{\dagger} A_{Q, 1} e^{-i Q d}+\right. \\
& \left.\gamma\left(A_{Q, n_{A}}^{\dagger} B_{-Q, 1}^{\dagger}+B_{Q, n_{B}}^{\dagger} A_{-Q, 1}^{\dagger} e^{-i Q d}\right)+h . c .\right]+N \frac{\left(n_{A} h_{A}+n_{B} h_{B}\left(f_{0 .}\right)\right)}{2}
\end{aligned}
$$

We can also write $H$ in the form 


$$
H=\sum_{Q} V_{Q}^{\dagger} \mathbf{T}(Q) V_{Q}
$$

where

$$
V_{Q}^{\dagger}=\left(A_{Q, 1}^{\dagger}, \ldots, A_{Q, n_{A}}^{\dagger}, B_{Q, 1}^{\dagger}, \ldots, B_{Q, n_{B}}^{\dagger}, A_{-Q, 1}, \ldots, A_{-Q, n_{A}}, B_{-Q, 1}, \ldots, B_{-Q, n_{B}},\right)
$$

and $\mathbf{T}(Q)$ is a matrix of dimension $2 d \times 2 d$ which represents the quadratic Hamiltonian $H_{Q}$. Since $\left[H_{Q 1}, H_{Q 2}\right]=0$, the diagonalization of the Hamiltonian is reduced to the diagonalization of $H_{Q}$, which can be written as a free fermion system

$$
H_{Q}=\sum_{k} E_{Q k} \xi_{Q, k}^{\dagger} \xi_{Q, k}
$$

where $E_{Q k}$ are the diagonal elements of $U_{Q} \mathbf{T}_{Q} U_{Q}^{\dagger}$, and $U_{Q}$ is the unitary transformation which diagonalizes $H_{Q}$, which is determined numerically. Since we are in the particle-hole representation, the energy spectrum is restricted to the positive solutions, and this means that it presents $d$ branches. By using the unitary transformation $U_{Q}$, we can express the fermion operators a's and b's in terms of the $\xi$ 's. This allows us to obtain immediately the induced magnetization per site and cell, which is defined as ${ }^{1}$

$$
\left\langle S_{c e l}^{z}\right\rangle=\frac{1}{N\left(n_{A}+n_{B}\right)}\left[\sum_{l=1}^{N}\left(\sum_{m=1}^{n_{A}}\left\langle S_{l, m}^{A^{z}}\right\rangle+\sum_{m=1}^{n_{B}}\left\langle S_{l, m}^{B^{z}}\right\rangle\right)\right],
$$

where

$$
\left\langle S_{l, m}^{A^{z}}\right\rangle=\left\langle a_{l, m}^{\dagger} a_{l, m}\right\rangle-\frac{1}{2}, \quad\left\langle S_{l, m}^{B^{z}}\right\rangle=\left\langle b_{l, m}^{\dagger} b_{l, m}\right\rangle-\frac{1}{2},
$$

which, at $T=0$, can be written in the form

$$
\left\langle S_{c e l}^{z}\right\rangle=\frac{1}{N\left(n_{A}+n_{B}\right)}\left[\sum_{m=1}^{n_{A}+n_{B}} \sum_{Q} \sum_{k\left(E_{Q k}>0\right)} u_{Q, k m}^{*} u_{Q, k m}\right]-\frac{1}{2}
$$

where $u_{Q, k m}$ are the matrix elements of the unitary transformation $U_{Q}$. 
By making the identification $h_{A} \equiv h$ and $h_{B} \equiv \alpha h$, the susceptibility $\chi^{z z}$, can be obtained immediately from the previous expression through the relation $\chi^{z z}=\frac{d}{d h}\left\langle S_{\text {cel }}^{z}\right\rangle$, which is determined numerically.

As an application of the results obtained, we have considered the case $n_{A}=n_{B}=2$ where the spectrum presents four branches. For $J=1$, $J_{A}=0.8, J_{B}=0.7, \gamma=0.1, \gamma_{A}=\gamma_{B}=0.2$ and $\alpha=2$, there are four critical modes (vanish at $Q d=0$ or $Q d=\pi$ ), which define the four critical fields $h_{c 1} \cong 0.01055, h_{c 2} \cong 0.36506, h_{c 3} \cong 0.54579$ and $h_{c 4} \cong 0.65604$. The spectrum, as in the isotropic model ${ }^{1}$, is numerically identical to the one obtained by using the transfer matrix approximation ${ }^{4}$. It all should be noted that, differently from the isotropic model ${ }^{1}$, the number of critical fields depends on the number of branches of the spectrum and on the interaction parameters.

Since, in this case, there are four critical fields, we have four quantum transitions induced by the transverse field, which are characterized by the divergences of the susceptibility. This result is shown in Figure 1, where the induced magnetization and the susceptibility, at $T=0$, are presented as a function of $h$. Although for this case, the divergences of the susceptibility are logarithmic $\left(\log \left|h-h_{c}\right|\right)$, it is possible to show, for special sets of parameters, that it can diverges as $\left|h-h_{c}\right|^{-1 / 2}$. This implies that the model on the alternating superlattice presents a parameter-dependent critical behaviour, and belongs to the same universality class of the model on the alternating chain ${ }^{5}$.

Acknowledgements. This work was partially financed by the Brazilian agencies CNPq and Finep.

\section{References}

[1] J. P. de Lima and L. L. Gonçalves, J. Magn. Magn. Mater., 206, 135 (1999)

[2] H. E. Lieb, T. Schultz and D. C. Mattis, Ann. Phys. 16, 407 (1961)

[3] L. L. Gonçalves and J. P. de Lima, J. Magn. Magn. Mater., 140 144, 1606 (1995)

[4] L. L. Gonçalves and J. P. de Lima, J. Phys.: Condens. Matter, 9, 3447 (1997)

[5] J. H. H. Perk, H. W. Capel, and M. J. Zuilhof, Physica 81A, 319 (1979)

\section{Figure captions.}


Figure 1. Induced magnetization (continous line) and the susceptibility $\chi^{z z}$ (dotted line), for $n_{A}=n_{B}=2, J=1, J_{A}=0.8, J_{B}=0.7, \gamma=0.1$, $\gamma_{A}=\gamma_{B}=0.2, \alpha=2$, at $T=0$,as a function of $h\left(h_{c 1} \cong 0.01055, h_{c 2} \cong\right.$ $0.36506, h_{c 3} \cong 0.54579$ and $\left.h_{c 4} \cong 0.65604\right)$. 


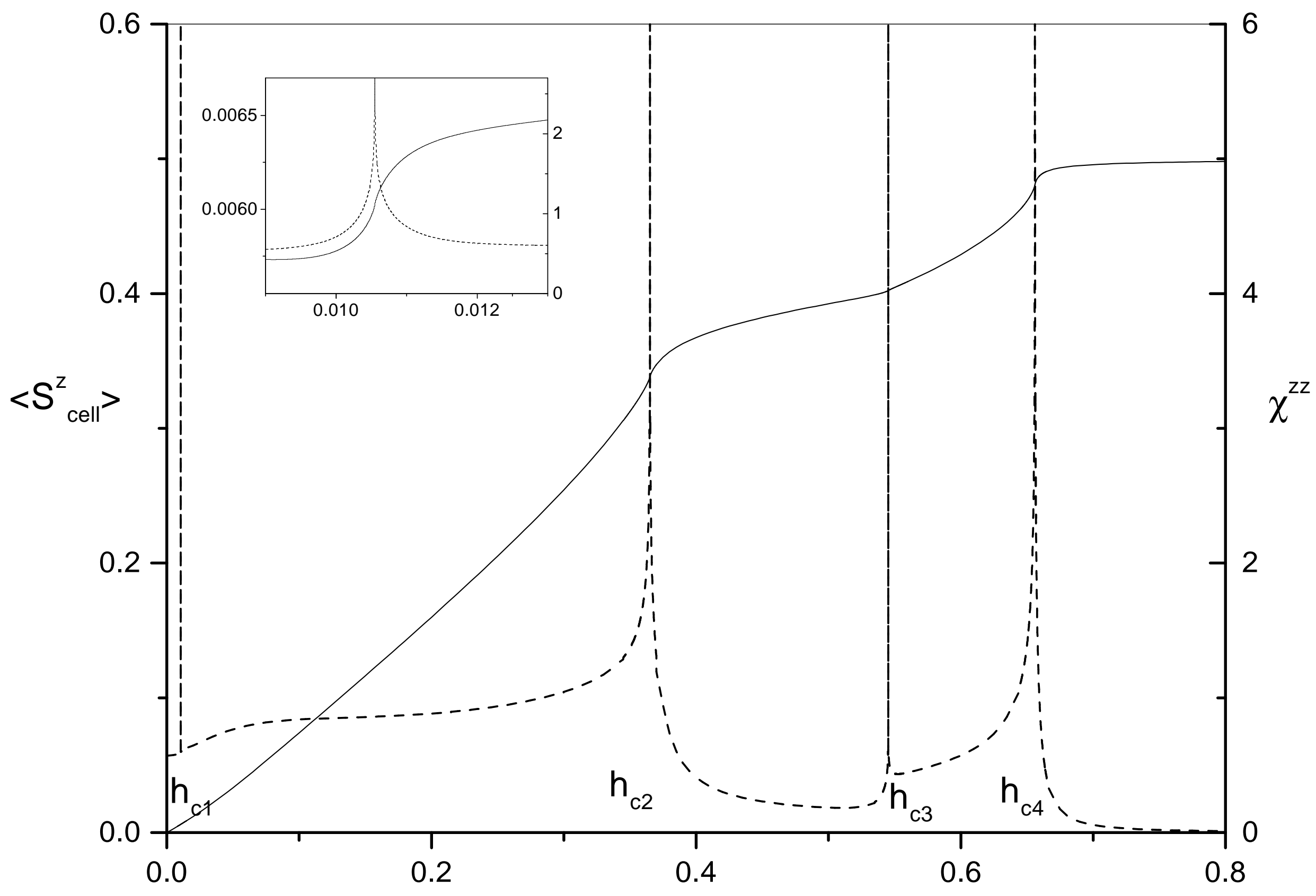

